\title{
Nominees elected to the Fellowship and Membership under Bye-Law III 2(ii)
}

At the meeting of the Court of Electors held on 16 February 1999, the following applicants were approved:

\section{The Fellowship}

Dr C. E. A. Adams, Dr. W. Ahmed, Dr R. W. Atkinson, Dr V. Bapuji Rao, Dr M. Bhate, Dr N. S. Brown, Dr T. M. Brown, Dr J. M. Burgess, Dr J. G. Byrne, Dr J. S. Callender, Dr C. A. D. Calvert, Dr D. V. Carpy, Dr R. Cawthra, Dr E. Chan-Pensley, Dr S. Chhabra, Dr C. J. Churcher-Brown, Dr M. Conway, Dr L. B. Cooke, Dr S. Cooray, Prof D. J. Cottrell, Dr C. A. Cruickshank, Dr H. A. Dickinson, Prof C. Duggan, Dr C. J. Fisher, Dr B. Fleming, Dr E. Fombonne, Dr S. B. Francis, Dr J. C. R. Galloway, Dr J. M. Green, Dr H. W. Griffiths, Dr P. H. Hollis, Dr P. Hopwood, Dr M. Ilo, Dr S. J. Johnston, Dr J. R. Jones, Dr S. B. Jones, Dr. J. Josse, Dr K. Kumar, Dr R. Kumar, Prof B. A. Lawlor, Dr C. A. R. Lind, Dr M. P. Lindsey, Prof R. M. Littlewood, Dr S. J. Logsdail, Dr S. S. Mahadeshwar, Dr B. Mann, Dr A. McFadyen, Dr G. J. McGrath, Dr G. C. Mezey, Dr S. F. Mitchell,
Prof A. M. Mortimer, Dr L. D. Mulgirigama, Dr J. M. Mullin, Dr J. S. Myles, Dr S. Narayan, Dr J. G. B. Newson-Smith, Dr M. C. O'Donovan, Dr A. Prabhakar, Dr D. Rajapakse, Dr A. C. Read, Dr E. D. Rice, Dr G. A. Roberts, Dr J. Roberts, Dr C. B. Sarathchandra, Dr T. D. Simpson, Dr D. M. St Clair, Dr R. A. D. Sykes, Dr C. S. Thomas, Dr C. A. Trotter, Dr P. J. W. van Boxel, Dr G. E. P. Vincenti, Dr L. J. Watt.

\section{Fellowships - overseas}

Dr S. A. Brooks, Dr P. M. Graham, Dr S. G. Kong, Dr S. Lee, Dr T. Mahmood, Dr S. B. Malik, Dr T. C. Ong, Dr J. H. Orley, Dr D. S. Samarasinghe.

\section{The Membership}

It was agreed that the following should be awarded Membership under Bye-Law III 2(ii): Prof C. Allwood, Prof Z. Bishry, Dr O. Gureje, Dr S. Khanna, Prof S. Priebe, Prof D. Somasundaram, Dr G. Ungvari.

\section{Winter Business Meeting 1999}

\section{Anne Dean}

The Winter Business Meeting of Council was held at the Royal College of Psychiatrists on 3 February 1999.

There were 17 Members present at this Meeting.

(a) Minutes

The Minutes of the Winter Business Meeting held at the Royal Lancaster Hotel, London on 21 January 1998 were approved as a correct record. (b) Election of Honorary Fellows

The following were elected to the Honorary Fellowship: Prof Charles Anthony Butterworth, Dr Jerome H. Jaffe, Prof William Alwyn Lishman, Baron Rix of Whitehall and City of Westminster and Prof Robert Anderson Wood.

Anne Dean, Head of Postgraduate Educational Services, Royal College of Psychiatrists, 17 Belgrave Square, London SWIX 8PG 